\title{
LASER DESORPTION DYNAMICS OF CARBENDAZIM ON A GLASS SURFACE
}

\author{
B. BESCÓS, J. M. OREA and A. GONZÁLEZ UREÑA* \\ Unidad de Láseres y Haces Moleculares, Instituto Pluridisciplinar, \\ Universidad Complutense de Madrid, Juan XXIII-1², 28040-Madrid, Spain
}

(Received 1 May 2000)

A study on the desorption of carbendazim (methyl-2-benzimidazolecarbamate, mass = $191 \mathrm{amu}$ ) from a glass surface (Pyrex) has been carried out using an experimental set-up based on laser desorption and laser post-ionization followed by time-of-flight mass spectrometry. After optimization of the experimental conditions a resolution of ca. 850 was achieved at the mass of the pesticide (191 amu). All measured time-of-flight mass spectra showed not only an excellent signal to noise ratio, but also the lack of significant molecular fragmentation, except for a mass of $71 \mathrm{amu}$ which was attributed to the lateral chain of the carbendazim. Neutral time-of-flight yields were measured for several desorption energies. Analysis of the corresponding velocity distributions suggests a thermal mechanism dominating the laser-induced desorption. Finally the capability of the present technique for analytical applications is also discussed.

Keywords: Desorption dynamics; Laser desorption; Laser ionization; REMPI; Time-offlight mass spectrometry; Carbendazim

\section{INTRODUCTION}

It is well known that the application of laser techniques in desorption are used increasingly for mass spectroscopy of solids either for analytical chemistry [1-7] or for increasing our basic understanding about surface science [8-14]. Of major relevance is the use of pulsed lasers to heat the adsorbate/surface system as it has shown a considerable advantage compared to other more conventional methods

\footnotetext{
*Corresponding author. Tel.: (+) 349139432 60, Fax: (+) 349139432 65, e-mail: laseres@eucmax.sim.ucm.es
} 
like temperature programmed desorption. For example, the development of laser-induced thermal desorption (LITD) provides a better method to combine pulsed dosing of the substrate and synchronized probing with tunable lasers. In this direction an important advance was implemented when one combines resonant-enhanced multiphoton ionization (REMPI) with time-of-flight mass spectrometry (TOFMS) using a pulsed laser as the probe tool [15-21]. From the fundamental point of view a major application of such techniques deals with the study of desorption dynamics. Indeed the enhanced sensitivity and resolution of these laser techniques provides an excellent method to get insight into the state-resolved internal energy distribution as well as into the translational energy distribution of desorbed molecules $[16,22-24]$. Thus, the fact that desorption and ionisation processes are separated in both space and time allows their specific study and optimisation. For example (see Results and Discussion Section) monitoring the desorption yield at the same ionisation region, but at different delay times with respect to the vaporisation laser, yields the velocity distribution of desorbed neutrals.

On the other hand, the application of these methods to the analysis of trace substances in gas mixtures or solid materials is perhaps one of the most exciting developments in modern chemical analysis. For the latter, one of the most important applications is the analysis of labile molecules. In this case fast desorption dominates over thermal decomposition making the analysis possible as the fast laser heating of the surface enables the observation of the mass spectrum of the intact parent ion [25-27].

In the present work we report on a study of the desorption of carbendazim (methyl-2-benzimidazolecarbamate, mass $=191 \mathrm{amu}$ ) from a glass surface (Pyrex). To implement such a study an experimental apparatus based on laser desorption and laser post-ionization followed by time-of-flight mass spectrometry (LD-LI-TOFMS) was designed and set up in our Molecular Beam and Laser Department [28]. Therefore, a clear feature of the present technique is the separation of both desorption and ionisation processes. This is an important advantage with respect to the more conventional MatrixAssisted Laser Desorption/Ionisation (MALDI technique) in which the two above processes cannot be separated [29].

A somewhat similar experimental design based on laser desorption has been reported for the investigation of fragile biomolecules via laser 
ionisation following the desorption process [30]. Nevertheless, these studies were accomplished by mixing the analyte with an excess of a chromophore molecule (matrix). Thus, from an experimental point of view the method requires the formation of a thin film containing a dye molecule and the analyte of interest. Here, in the present technique and investigation, such a requirement is nolonger needed as the analyte is used directly.

Not only has the study of the fundamental aspects of carbendazim desorption motivated the present work, but specially the fact that this molecule is currently used as one of the pesticides in agriculture, particularly in the south of Spain [31]. In consequence, the development of a fast and high sensitivity analytical method is shown to be of major importance for practical diagnosis and quality control of agricultural samples.

\section{EXPERIMENTAL SET-UP}

The experimental set-up consists of two stainless steel ultrahigh vacuum chambers designed and built in our Institute. The first chamber is used for both laser desorption and laser post-ionization of the sample. This is followed by ion acceleration towards the second chamber, which is basically the time-of-flight (TOF) unit including accelerating plates, deflectors, lenses and a double microchannel plate detector. Figure 1 shows a schematic layout of the set-up. The two chambers can be pumped independently by their respective turbomolecular plus rotary pumps units, namely: a Varian V250 plus Telstar RD 18 for the first chamber, and a Varian V70 plus a Telstar 2P-3 for the second one. A gate valve maintains the second chamber pressure below $5 \cdot 10^{-8}$ mbar even when the first chamber was open for sample manipulation. The pressure of the two chambers was always below $10^{-6}$ mbar during experiments.

The inset of Figure 1 shows a detailed view of the first ionization chamber for a better illustration of the experimental basis of the technique. The analyte is deposited by spraying the solution on a $75 \mathrm{~mm}$ pyrex disc which is parallel to the first accelerating plate $(V 1)$ and $2 \mathrm{~mm}$ from it. A step motor (Crouzet) is used to control the disc rotation. The whole sample holder and motor assembly is attached to a linear translator so that adjustment of the $Z$ distance (i.e., the 


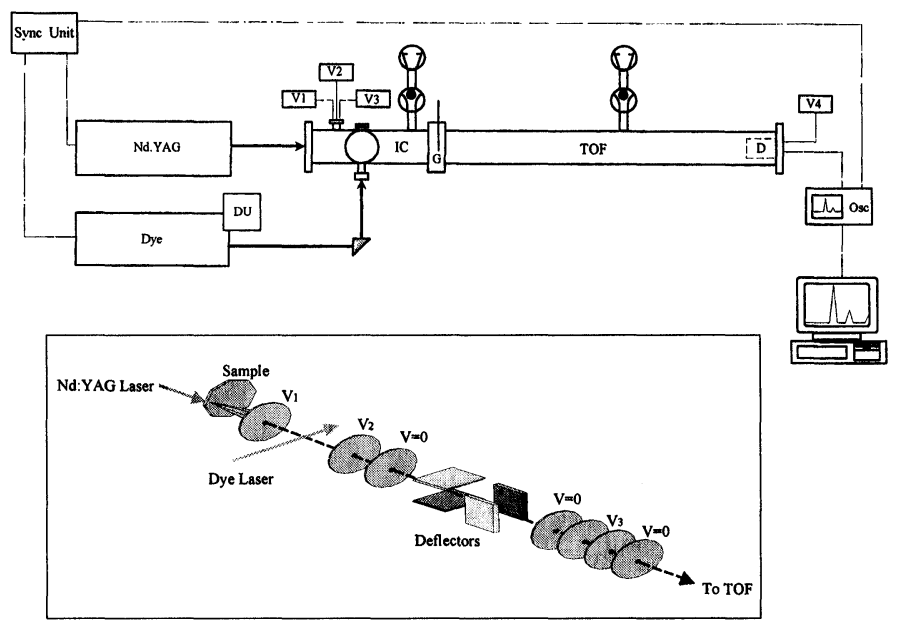

FIGURE 1 Experimental setup. The inset shows the internal parts of the ionization chamber. IC stands for the ionization chamber, TOF for the time-of-flight tube and SU for the synchronisation unit between both lasers ( $L 1$ and $L 2) . V 1$ and $V 2$ are the accelerating voltages, $V 3$ the ionic lens voltage, and $V 4$ the microchannel plates detector (D) voltage. $T$ and $R$ stand for both turbomolecular and rotary pumps respectively.

perpendicular direction to the desorption laser) can be made. This movement changes the rotation radius so as to increase the number of runs for a given sample.

Pulses of $5 \mathrm{~ns}$ of duration of the second harmonic of a Nd:YAG laser (Continuum) were used for sample desorption. The laser beam was steered by prisms and directed to the rear of the sample disc without focussing. Laser desorption energies used in the present work ranged from 15 up to $75 \mathrm{~mJ} / \mathrm{cm}^{2}$. The (pulsed) desorbed neutrals were expanded into the acceleration region and, subsequently, ionized by the 4th harmonic of a second Nd:YAG laser (Continuum ND81). For some experiments a frequency-doubled dye laser (Continuum ND 60) was also used to ionize the desorbed neutrals. The ionization laser was always perpendicular to that of desorption and crossed the desorbed particles at a variable distance ranged from 6 up to $30 \mathrm{~mm}$ apart from the desorption zone.

The ions produced by the second laser were then accelerated towards the TOF tube. The acceleration unit consists of a system of high voltage plates working in two steps (i.e., $V_{1} \rightarrow V_{2}, V_{2} \rightarrow V_{3}=0$ ). In addition, the system includes an Einzel lens as well as two deflecting 
plates positioned along the $X$ and $Y$ directions and therefore perpendicular to $Z$, that is the TOF axis.

To obtain the time-of-flight spectrum a two microchannel plate detector (Comstock CP-625C/50F) was used and its output signal was fed into a digital scope (Tektronix 540) that averages 100 laser shots. The TOF spectrum was then transferred to a computer for storage and further analysis.

The delay between the desorption and ionization laser pulses was controlled by a pulse generator (Lyons Instruments). In all experiments the laser repetition frequency was $10 \mathrm{~Hz}$. Laser pulse energies were monitored either by a calorimetric detector (Photon Control 25) for high energies $(10-40 \mathrm{~mJ})$ or by a pyroelectric detector (Gentec Ed$100 \mathrm{~A})$ for low pulse energies $(0-10 \mathrm{~mJ})$. For the present work the size of both desorption and ionization volumes was found to be crucial. Consequently, variable diaphragms were incorporated in the system. Finally, for the calibration experiments in which a gas sample was used, the entire desorption unit was replaced by a gas inlet and obviously only the ionization laser was used.

\section{RESULTS AND DISCUSSION}

\section{Mass Calibration and Time-of-flight Spectrum}

To calibrate the apparatus a preliminary experiment was carried out introducing a gas mixture of toluene and aniline which were ionized using $2 \mathrm{~mJ}$ pulses of the $266 \mathrm{~nm}$ laser. The choice of toluene and aniline was based not only on their well known multiphoton ionization spectra [32], but basically on the fact that their masses differ by one $\mathrm{amu}$. The test was fully satisfactory indicating a good mass resolution as described below. With the experimental conditions optimized, laser desorbed carbendazim vapour was introduced in the ionization chamber resulting in the time-of-flight spectrum displayed in Figure 2. Notice not only the excellent signal-to-noise ratio, but also the lack of significant fragments yield, except for a fragment of mass $71 \mathrm{amu}$ which can be attributed to the lateral chain of the carbendazim (see below). A resolution $R=t / 2 \cdot(\Delta t)_{\mathrm{FWHM}}=834$ was determined, as can be deduced from the bottom of the figure, at the mass of this 

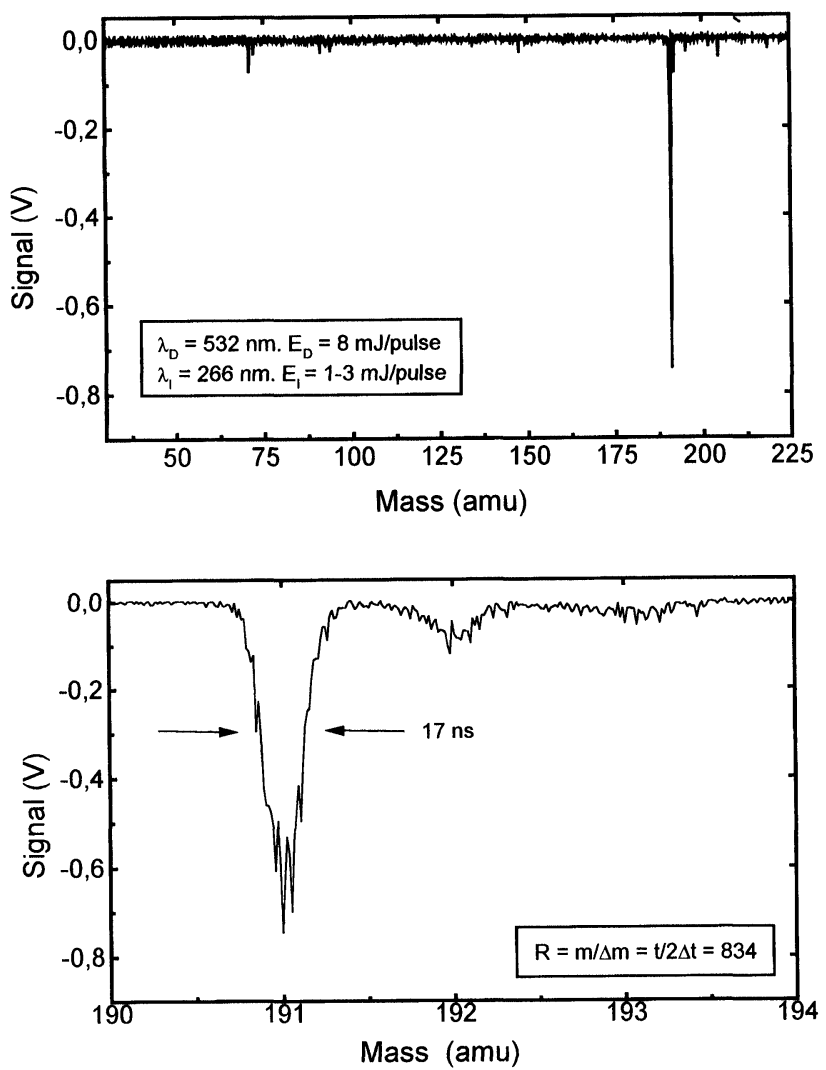

FIGURE 2 Top: Carbendazim TOF mass spectrum obtained by MPI. Bottom: Typical resolution obtained for carbendazim $(R=834$ for mass $=191 \mathrm{amu})$.

pesticide $(191 \mathrm{amu})$. The relevant experimental conditions of the carbendazim ionization are listed in Table I, together with other experimental features of the technique.

\section{Ionization Dependence on Laser Energy}

Figure 3 shows the dependence of the carbendazim ion signal on the laser energy at a fixed wavelength of $281.5 \mathrm{~nm}$. A clear signal enhancement with increasing laser energy can be noticed. This energy dependence is more clearly displayed in the upper part of Figure 4 in which a $\log -\log$ plot of the data suggests that the carbendazim is 


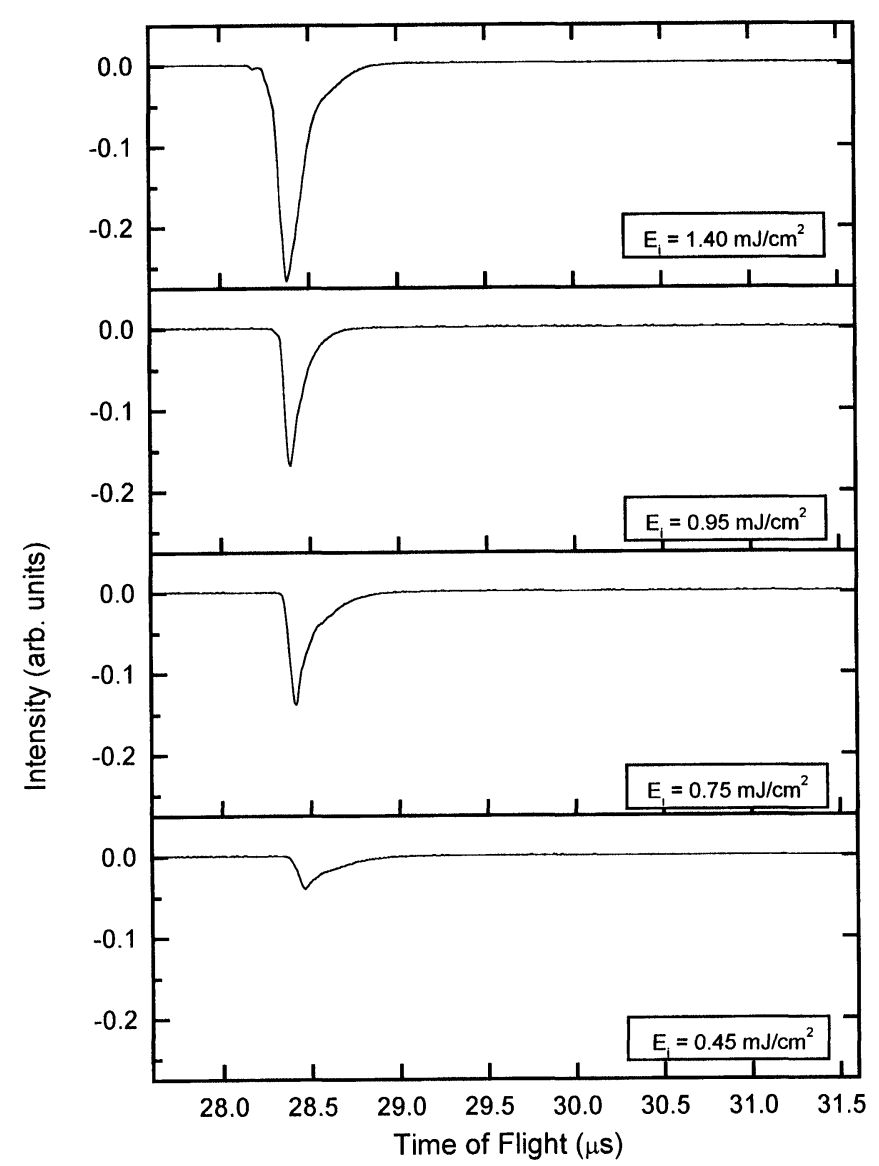

FIGURE 3 Carbendazim TOF spectra as a function of the ionization energy at constant desorption energy of $40 \mathrm{~mJ} / \mathrm{cm}^{2}$. Ionization laser wavelength $\lambda_{i}=281.5 \mathrm{~nm}$.

experiment was repeated at different delay times between desorption and ionization to take into account variations in the initial kinetic energy of the desorbed molecules with increasing laser desorption power. The amount of signal was always decreasing when increasing the energy, indicating fragmentation of the sample in the desorbing process. Further evidence of it was the presence of new fragments in the spectrum. The pronounced decline in the desorption yield of the carbendazim beyond $40 \mathrm{~mJ} / \mathrm{cm}^{2}$ can be attributed to the onset of molecular fragmentation as the laser desorption fluence increases. 

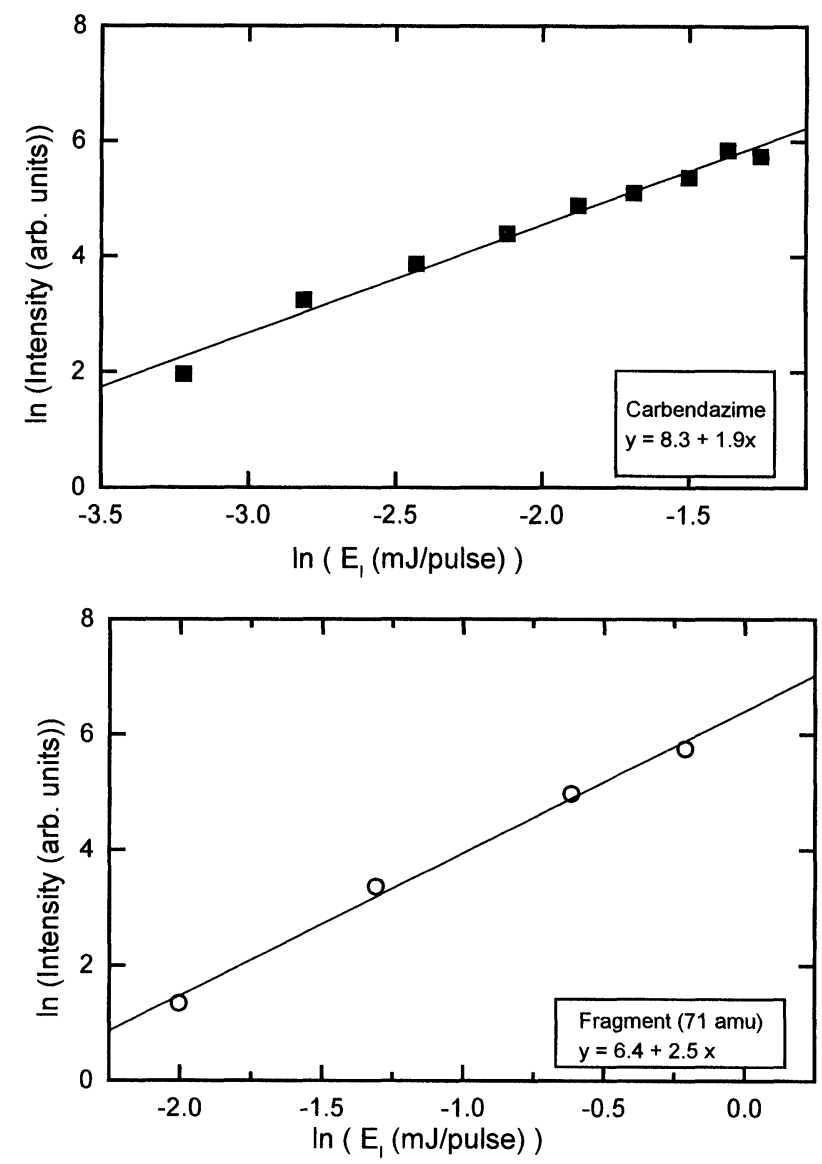

FIGURE 4 Logarithmic plot of the intensity of the parent ion (Top) and fragment $(71 \mathrm{amu})$ (Bottom) signals $v$ s. ionization energy. Laser wavelength $=281.5 \mathrm{~nm}$.

\section{Desorption Mechanism: Velocity Distributions of Neutrals Ejected from the Crystal Surface}

In order to measure the velocity distribution of the ejected material TOF spectra were registered as a function of the delay between the two laser pulses. Knowing the distance between the desorption and ionization regions and the delay time, the initial speed of the desorbed species can be then calculated. Figure 7 shows the carbendazim TOF spectra as a function of the delay between both laser pulses. Since in 


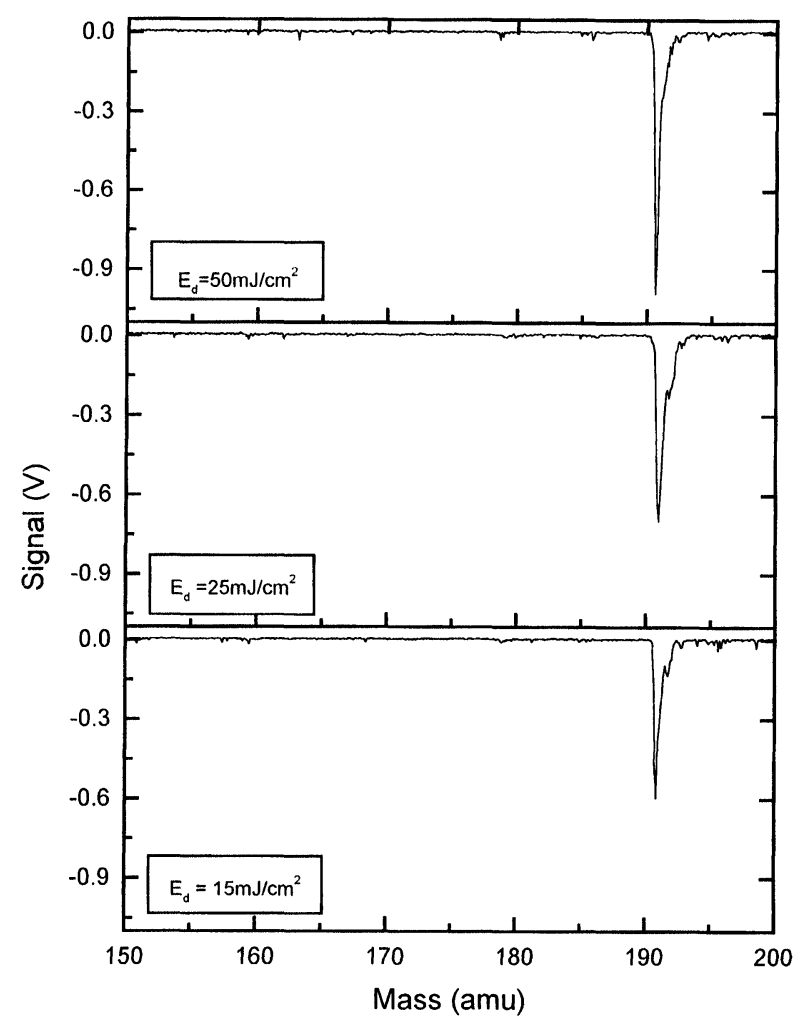

FIGURE 5 Carbendazim TOF spectra as a function of the desorption energy at constant ionization energy of $7.5 \mathrm{~mJ} / \mathrm{cm}^{2}$. $\left(\lambda_{\text {desorption }}=532 \mathrm{~nm}\right.$ and $\left.\lambda_{\text {ionization }}=266 \mathrm{~nm}\right)$.

our arrangement the main component of the velocity lies in the direction of the TOF axis, the initial velocity of the molecules being selected by the ionization pulse adds up to the velocity acquired due to the extracting field and the position of the signal in the time of arrival spectrum is shifted to longer times with increasing delay times (slower molecules).

Figure 8 shows the intensity of carbendazim signal as a function of its velocity for several desorption laser energies, namely 25, 50 and $75 \mathrm{~mJ} / \mathrm{cm}^{2}$. The most probable velocities were found to be 260,290 and $350 \mathrm{~m} / \mathrm{s}$ for the above desorption energies, respectively. The experimental velocity distributions were fitted by 


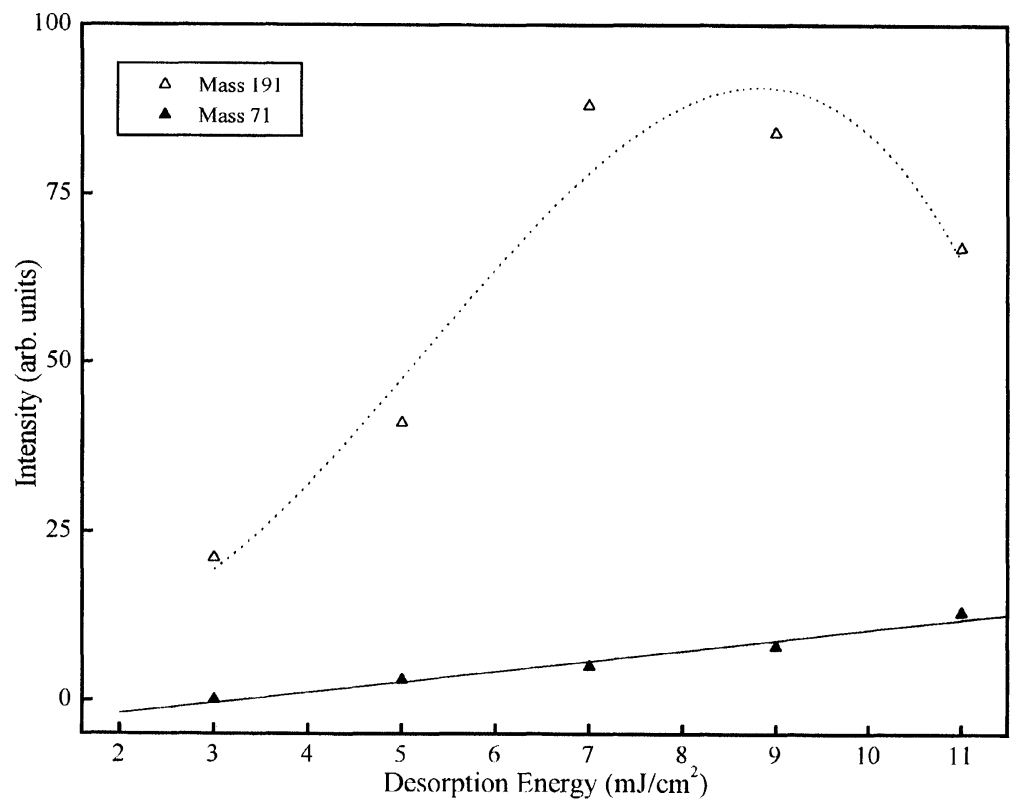

FIGURE 6 Intensity $v s$. desorption energy for carbendazim and fragment (71 amu).



FIGURE 7 Carbendazim TOF spectra as a function of the delay between the desorption laser and the ionization laser. 
Maxwell-Boltzmann distributions which are displayed in Figure 9. The resulting temperatures range from 790 up to $1500 \mathrm{~K}$ for desorption energies between 25 and $75 \mathrm{~mJ} / \mathrm{cm}^{2}$, respectively, as listed in Table II. The same type of velocity distributions are shown for the 71 amu fragment in Figure $8 \mathrm{~b}$. These velocity distributions correspond to similar temperatures as found for carbendazim. This is consistent with a local thermal equilibrium at the surface, dominating the desorption process.

Similar results to those reported here have been published in the literature for other organic compounds. For example, a study [22]



FIGURE 8 Intensity vs. velocity of the ions for different desorption energies. The dashed lines represent the maxwellian fit to the data. (a) Carbendazim. (b) Fragment (Mass $=71 \mathrm{amu}$ ). 


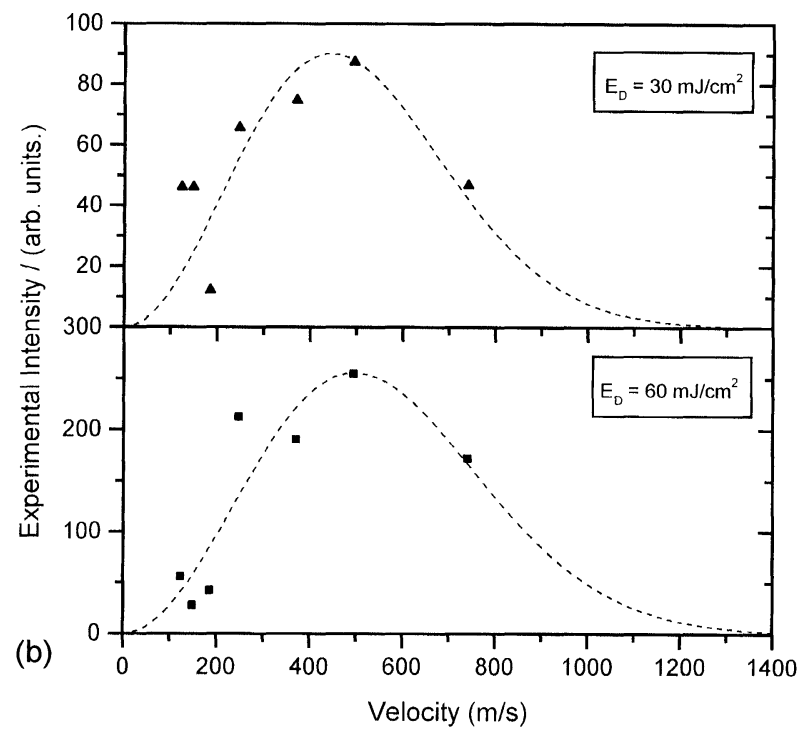

FIGURE 8 (Continued).

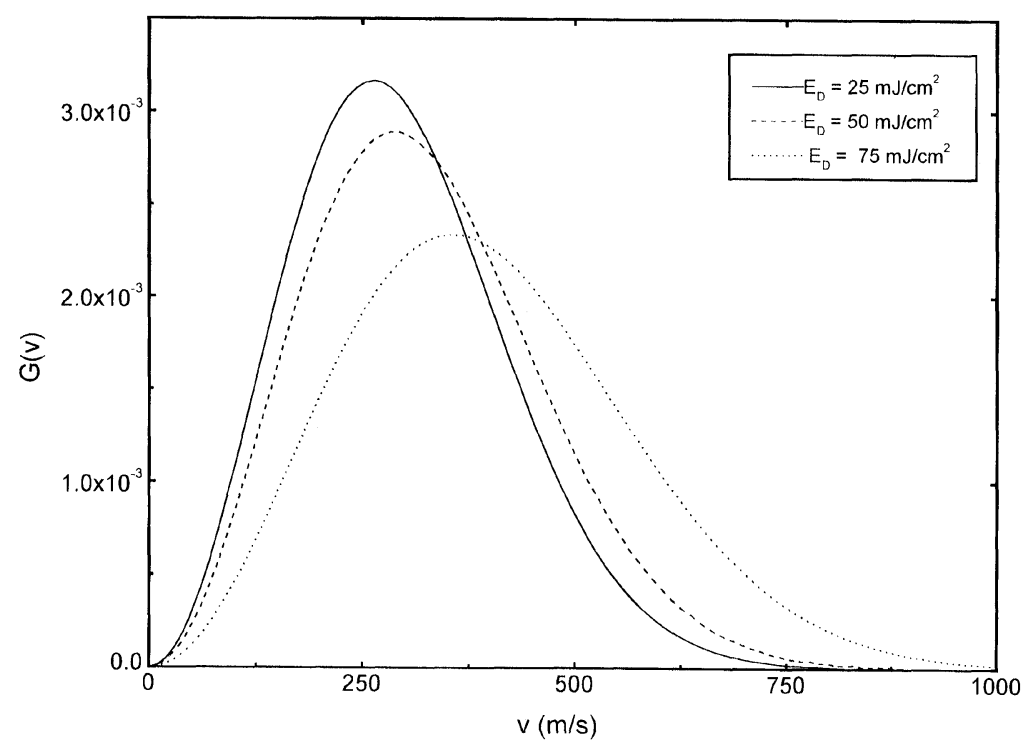

FIGURE 9 Evolution of the velocity energy distribution with the desorption energy for the carbendazim ions. 
TABLE II Temperature obtained from the kinetic energy distributions of the desorbed molecules at the different laser desorption energies

\begin{tabular}{lc}
\hline Desorption energy $\left(\mathrm{mJ} / \mathrm{cm}^{2}\right)$ & Temperature $(\mathrm{K})$ \\
\hline 25 & 791 \\
50 & 949 \\
75 & 1455 \\
\hline
\end{tabular}

on the desorption of aniline from a metal surface revealed that the velocity distribution of desorbed neutrals could be fitted by a Maxwell-Boltzmann distribution with a temperature of $700 \mathrm{~K}$. This study suggests a thermal mechanism dominating the desorption process. However the same translational temperature of the desorbed particle distribution was found for different laser desorption energies, the desorption yield being the major observed change with laser energy. In contrast our results indicate not only a substantial increase in the desorption yield of carbendazim, but also a linear increase of the translational energy of desorbed neutrals.

This trend can be expected from a substrate mediated thermal desorption mechanism. In other words, the carbendazim absorption plays a minor role on the desorption. This is not, in principle, surprising as the employed laser photon at $\lambda=532 \mathrm{~nm}$ is far from any absorption band of the carbendazim.

On the other hand, there are some studies in which bimodal (translational) energy distributions were found for neutral desorbed species, as for example in Rhodamine 6-G and anthracene [23] and NO [16]. In these experiments a very fast component followed by a slow (Maxwell-Boltzmann type) component were found. As far as the present results are concerned the fast component, if it exists, is a very minor component which could not be resolved under our experimental resolution.

Zhang et al. [24] investigated benzimidazole (a carbendazim like molecule) and a unique thermal velocity distribution was found. The average velocities calculated were slightly higher, but comparable to those of the present study. This could be a consequence of the lower mass of benzimidazole compared to that of carbendazim, when, as it is the case, the average translational energies they observed were very similar. An interesting aspect of that study was the finding of a cooling effect, i.e., a decrease in the internal energy 
of the desorbed neutrals, as the ionization region distance to the surface increased. In addition, the same authors found that this cooling effect also depends on the matrix used to support the sample. The observed trend was that the simpler the molecule forming the matrix the higher the cooling of the internal modes of the desorbed neutrals. In our experiments we did not use a matrix to facilitate this cooling. Furthermore the geometry of our design does not allow ionization beyond $30 \mathrm{~mm}$ from the Pyrex surface which may justify why our average velocity values are smaller than those of benzimidazole.

As mentioned above the time-of-flight spectrum of carbendazim under our experimental conditions, listed in Table I, shows two peaks corresponding to 191 and 71 a.m.u. The $191 \mathrm{amu}$ peak is that of carbendazim molecule and that of $71 \mathrm{amu}$ may probably originate from the lateral chain of the molecule after fragmentation.

The fragment is formed irrespective of the desorption laser wavelength (e.g., $1064 \mathrm{~nm}$ or $532 \mathrm{~nm}$ ). One could argue about the possibility that it is a sample impurity. However a HPLC analysis of the carbendazim resulted in a percent purity greater than $99,9 \%$. To account for the significant signal peak of the 71 mass one must then suppose a much lower ionization probability for the carbendazim than for the impurity and this hypothesis seems very unlikely. In addition, from Figure 4 one can observe that this fragment shows a higher order ionization process than carbendazim.

It is interesting to remark that both carbendazim and fragment velocity distributions can be described by similar temperatures. This is illustrated in Figure 10 in which the translational energy distributions are also shown at the bottom. The estimated temperatures for carbendazim and its $71 \mathrm{amu}$ mass fragment are $855 \mathrm{~K}$ and $825 \mathrm{~K}$, respectively for a desorption energy of $30 \mathrm{~mJ} / \mathrm{cm}^{2}$. When this is increased up to $60 \mathrm{~mJ} / \mathrm{cm}^{2}$ temperatures of $1160 \mathrm{~K}$ and $1070 \mathrm{~K}$ were then obtained, respectively.

This finding combined with the observation that both parent and fragment translational energies follow a similar trend with desorption energy suggest that a thermal mechanism is probably controlling the process. In addition it may also indicate that fragmentation occurs right at the desorption region. A possible fragmentation scheme could involve molecular rearrangement of the excited parent molecule after 
excitation by the desorption photon. Schematically:
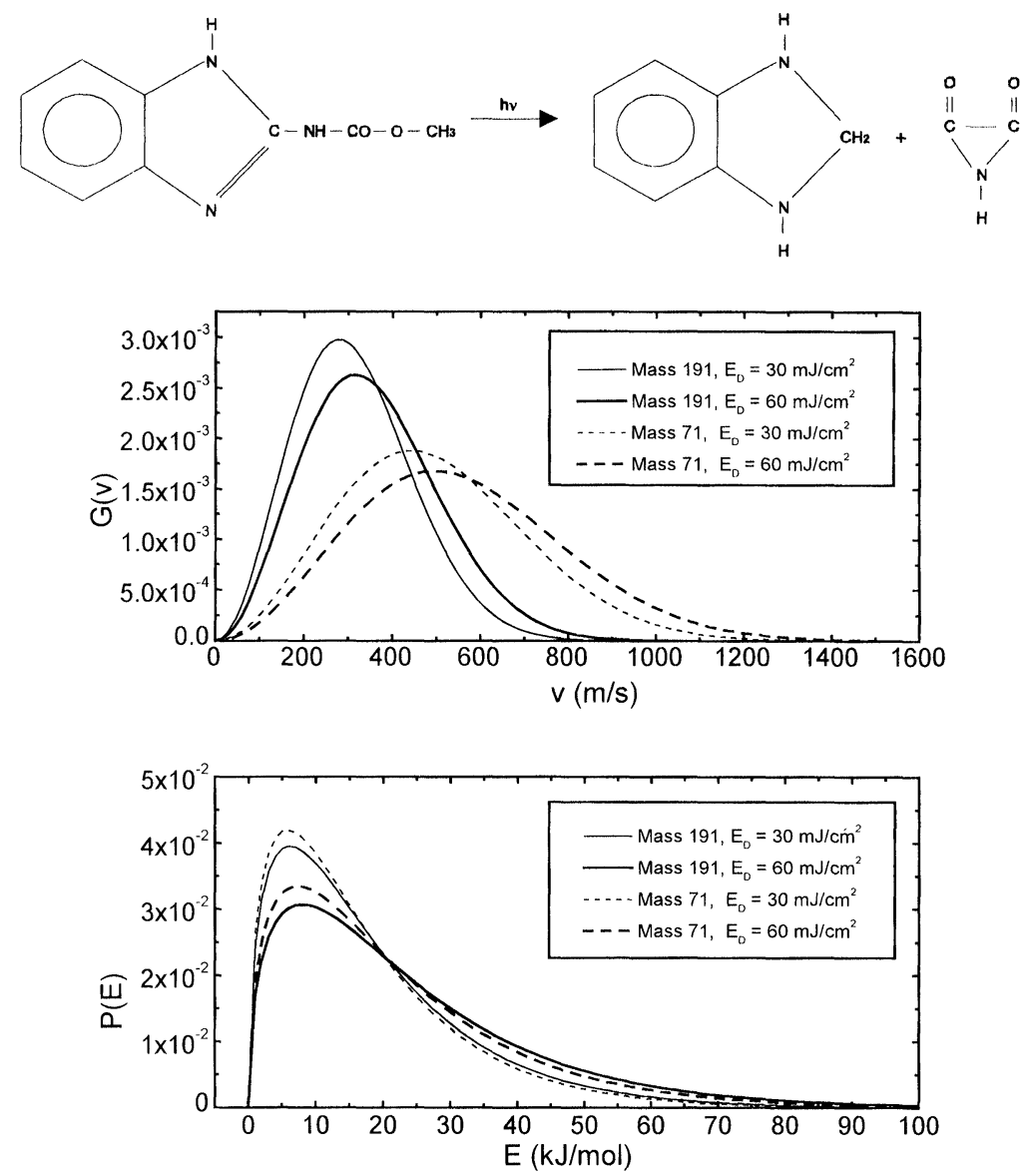

FIGURE 10 Top: Velocity distributions, $G(v)$, for carbendazim and fragment (71 amu) for different desorption energies. Bottom: Kinetic energy distributions, $P(E)$, for carbendazim and fragment (71 amu) for different desorption energies.

To reinforce the latter hypothesis, against that where fragmentation occurs during carbendazim ionization, several observations can be considered. Namely:

(a) Many fragments appear when the fluence of the ionization laser is increased. Both parent and fragment signal peaks are broad, however the 71 amu peak remains narrow. 
(b) If the $71 \mathrm{amu}$ fragment were produced during the ionization of the carbendazim vapour, the fragment velocity distribution would appear centered at the most probable velocity of the parent molecule. Thus assuming an isotropic distribution of the fragments the only consequence would be a broadening in the velocity distribution. Nevertheless the observation is against it, as the experimental distribution does not follow that pattern. In contrast, it shows higher velocities than those of the parent molecule, but, as already mentioned, with a similar mean translational energy.

(c) The observed desorption threshold for the fragment seems to be higher than for the carbendazim (see Fig. 6 for a better illustration).

\section{CONCLUDING REMARKS}

A technique based on laser desorption and laser ionization coupled with time-of-flight mass spectrometry has been set-up to investigate desorption processes of organic adsorbates. In particular, a study of carbendazim desorption has been carried out leading to the following conclusions:

(a) Carbendazim laser desorption at $532 \mathrm{~nm}$ seems to be an efficient process with minor fragmentation except for a $71 \mathrm{amu}$ fragment. The latter seems to be produced by molecular re-arrangement of the carbendazim lateral chain after molecular thermal excitation during the desorption process.

(b) Carbendazim ionization at $266-281.5 \mathrm{~nm}$ range seems to occur through a two-photon process with significant ion yield and little fragmentation.

(c) The velocity distributions of the desorbed neutrals can be well described assuming a thermal mechanism controlled by the surface temperature. In other words, the carbendazim absorption plays little role, the desorption yield being dominated by a thermal substrate-mediated mechanism.

Finally, the present technique is shown to be very promising in chemical analysis for environmental samples containing pesticides. This will be particularly interesting when the optimized resonant- 
enhanced-multiphoton ionization (REMPI) of such samples is available as it will lead to high sensitivities in their analysis. Work is in progress along this direction for analysis of solid materials, and specifically for agricultural samples. First results from this research activity has been the subject of a recent publication [33].

\section{Acknowledgements}

This work received financial support from the DGICYT of Spain grant number PB97/272 and the Comunidad Autónoma de Madrid.

\section{References}

[1] Montero, C., Bescós, B., Orea, J. M. and González Ureña, A. (2000). Rev. of Anal. Chem., XIX, 1-29.

[2] Mayo, S., Lucatorto, T. B. and Luther, G. G. (1982). Anal. Chem., 54, 553.

[3] Cotter, R. J. (1987). Anal. Chim. Acta., 195, 45.

[4] Heise, T. W. and Yeung, E. S. (1994). Anal. Chem., 66, 355.

[5] Nicolussi, G. K., Pellin, M. J., Calaway, W. F., Lewis, R. S., Davis, A. M., Amari, S. and Clayton, R. N. (1997). Anal. Chem., 69, 1140.

[6] Spengler, B. and Cotter, R. J. (1990). Anal. Chem., 62, 793.

[7] Castaño, J., Skowronek, S., Garay, M., Bescós, B., Zapata, V., Orea, J. M., Laplaza, A., Tardajos, G., Pereira, R., Rinaldi, C. and González Ureña, A. (1997). Laser Chem., 17, 29.

[8] Kimbrell, S. M. and Yeung, E. S. (1991). Appl. Spectrosc., 45(3), 442.

[9] Kinsel, G. R., Lindner, J., Grotenmeyer, J. and Schlag, E. W. (1991). J. Phys. Chem., 95, 7824.

[10] Karas, M., Bachmann, D. and Hillenkamp, F. (1985). Anal. Chem., 57, 2935.

[11] Heise, T. W. and Yeung, E. S. (1995). Anal. Chim. Acta., 299, 377.

[12] Weinkauf, R., Aicher, P., Wesley, G., Grotemeyer, J. and Schlag, E. W. (1994). J. Phys. Chem., 98(34), 8381.

[13] Spengler, B. and Kaufmann, R. (1992). Analysis, 20(3), 91.

[14] Vertes, A. and Levine, R. D. (1990). Chem. Phys. Letters, 71(4), 284.

[15] Zare, R. N. (1984). Science, 226, 298.

[16] Zimmermann, F. M. and Ho, W. (1994). J. Chem. Phys., 100(10), 7700.

[17] Boesl, U., Zimmermann, R., Weickhardt, C., Lenoir, D., Schramm, K. W., Kettrup, A. and Schlag, E. W. (1994). Chemosphere, 29(7), 1429.

[18] Boesl, U., Weinkauf, R. and Schlag, E. W. (1992). Int. J. Mass Spectrom. Ion Processes, 112, 121.

[19] Bernstein, E. R., Law, K. and Schauer, M. (1984). J. Chem. Phys., 80(1), 207.

[20] Zhang, L., Pei, L., Dai, J., Zhang, T., Chen, C., Yu, S. and Ma, X. (1996). Chem. Phys. Letters, 259, 403.

[21] Weickhardt, C., Boels, U. and Schlag, E. W. (1994). Anal. Chem., 66, 1062.

[22] Yang, M. and Reilly, J. P. (1990). J. Phys. Chem., 94, 6299.

[23] Srinivasan, J. R., Romano, L. J. and Levis, R. J. (1995). J. Phys. Chem., 99, 13272.

[24] Zhang, J. Y., Nagra, D. S. and Li, L. (1993). Anal. Chem., 65, 2812.

[25] Heise, T. W. and Yeung, E. S. (1992). Anal. Chem., 64, 2175.

[26] Bagheri, H. and Creaser, S. J. (1991). Cromatogr., 547, 345. 
[27] Li, L. and Lubman, D. (1988). Rev. Sci. Instrum., 59(4), 557.

[28] Bescós, B., Doctoral Thesis, Universidad Complutense, Madrid, March, 1997.

[29] Karas, M., Bachmann, D., Bahr, U. and Hillenkamp, F. (1987). J. Mass Spec. Ion Processes, 78, 53.

[30] Schilke, D. E. and Levis, R. J. (1994). Rev. Sci. Intrum., 65(6), 1903.

[31] Aguera, A., Contreras, M. and Fernández-Alba, A. R. (1993). J. Chromatogr. A, 655, 293.

[32] Lubman, D. M. and Kronick, M. N. (1982). Anal. Chem., 54, 660.

[33] Orea, J. M., Bescós, B., Montero, C. and González Ureña, A. (1998). Anal. Chem., 70, 491. 